\title{
Feasibility of a TPS-integrated method to incorporate tumor motion in the margin recipe
}

David Dechambre ${ }^{1}$, Loïc Vander Veken ${ }^{2}$, Antoine Delor ${ }^{1}$, Edmond Sterpin $^{2,3}$, Françoise Vanneste ${ }^{1}$, Xavier Geets ${ }^{1}$

${ }^{1}$ Cliniques Universitaires Saint-Luc, Radiotherapy department, Brussels, Belgium

${ }^{2}$ Université catholique de Louvain, Institut de Recherche Expérimentale et Clinique (IREC), Center of Molecular Imaging, Radiotherapy and Oncology (MIRO), Brussels, Belgium

${ }^{3} \mathrm{KU}$ Leuven, Department of Oncology, Laboratory of Experimental Radiotherapy, Leuven, Belgium

Corresponding author:

David Dechambre, M.S.

Radiotherapy department

Cliniques Universitaires Saint-Luc

10 Avenue Hippocrate

1200 Brussels, Belgium

david.dechambre@uclouvain.be

$+32491743909$ 


\section{Abstract}

Background and Purpose: There are several alternatives to the widespread ITV strategy in order to account for breathing-induced motion in PTV margins. The most sophisticated one includes the generation of a motion-compensated CT scan with the CTV placed in its average position - the midposition approach (MidP). In such configuration, PTV margins integrate breathing as another random error. Despite overall irradiated volume reduction, such approach is barely used in clinical practice because of its dependence to deformable registration and its unavailability in commercial treatment planning systems. As an alternative, the mid-ventilation approach (MidV) selects the phase in the 4DCT scan that is the closest to the MidP, with a residual error accounted for in the PTV margin. We propose a treatment planning system-integrated strategy, aiming at better approximating the MidP approach without its drawbacks: Hybrid MidV-MidP approach, i.e. the delineation on the MidV-CT and translation at the mid-position coordinates using treatment planning system built-in capabilities.

Material and Methods: Forty-five lung lesions treated with stereotactic radiotherapy were selected. PTV was defined using MidP, MidV, Hybrid MidV-MidP and ITV strategies. Margin definitions were adapted and resulting PTVs were compared.

Results: Hybrid MidV-MidP showed similar target volume and location than the MidP and confirmed that margin-incorporated tumor motion strategies lead to significantly smaller PTVs than the ITV with mean reduction of $26 \pm 7 \%$.

Conclusion: We report on the successful implementation of a pseudo-MidP solution without its inherent drawbacks. It answers the need for TPS-embedded tumor motion range identification and related margin's component calculation.

Keywords: motion management; mid-position; mid-ventilation; 4DCT; margin recipe 


\section{Introduction}

In radiotherapy, reduced toxicity levels and improved treatment outcome can be obtained by limiting the treatment volume that receives curative doses. This can be pursued by reducing the planning target volume (PTV) margins. The PTV is generally defined as the clinical target volume (CTV: visible tumor plus a margin for suspected microscopic extensions) plus a margin to account for geometric uncertainties[1]. These uncertainties are due to inter-fractional geometric uncertainties related to patient setup errors and respiratory baseline shifts (i.e. shifts in average tumor position over time) but also largely due to intra-fractional uncertainties related to respiratory motion[2]. As such, minimizing the latter will translate into a reduction of the CTV to PTV margins, and thus a reduction in the irradiated normal tissue volume. This is of particular importance for lung stereotactic body radiotherapy (SBRT) where surrounding organs might limit the tumor dose in risk-based prescription schemes[3].

To address this problematic, time-resolved four-dimensional (4D) scanning technique was developed. It consists in the acquisition of oversampled three-dimensional (3D) data that are sorted, most of the time, by the patient's respiratory phase using an external breathing signal [4-7]. This results in a 4D image data set composed of a set of 3D reconstructions at different breathing phases. It provides temporal and spatial motion information that can be used to optimize treatment planning. Practical applications of these planning system-based motion management technique are found in studies published by Engelsman et al [8] and Witte et al.[9]. The basic principle is to define the tumor at its time-weighted average position (mid-position) along the respiratory cycle. As a result, tumor motion can be incorporated in the CTV to PTV margin expansion as an additional random error. This is opposite to the widespread use of the internal target volume (ITV) that encompasses the entire tumor motion and therefore considers respiratory motion as a systematic error. Thanks to the large penumbra in the lung, irradiation of the tumor at its mid-position allows for good dose coverage even if the lesion is not fully within the high-dose region for a small part of the breathing cycle[8,10,11]. Based on this rationale, the mid-ventilation strategy (MidV) was developed[12]. It consists in defining the tumor on a single well-chosen phase of a 4D-CT where the lesion is the closest to its average position in the respiratory cycle. The mid-position strategy (MidP) is considered as a refined version of the MidV strategy[13]. The tumor is delineated on an artificial motioncompensated CT-scan presenting the tumor and the internal structures at their time-weighted average position during the respiratory cycle. By directly representing the tumor at its mean position, it removes the need to account for the residual distance between the MidV and MidP positions in the MidV margin recipe. Nevertheless, the generation of an additional CT is not convenient and the results are hardly verifiable due to the lack of deformable image registration (DIR) QA tools.

Despite the potential benefits of the MidV/MidP approaches, their deployment in clinical practice is hindered by the lack of commercial solutions providing an integrated approach to 4DCT treatment planning [10,14-16].

In this context, an alternate treatment planning system (TPS)-integrated solution was developed. Solving the issues of both MidP and MidV strategies, the Hybrid MidV-MidP approach consists in delineating the tumor on the 4D-CT MidV phase and translating it to the mid-position coordinates. 
The first aim of the study is to attest the feasibility of developing an entire TPS-integrated workflow dedicated to motion management. The second is to validate the benefits of using our novel mixed approach (i.e. the Hybrid MidV-MidP) by measuring its impact on margin amplitude and irradiated volume as compared to the MidV, MidP and the commonly used ITV strategies.

\section{Methods}

\section{Patient group}

Thirty-six patients, treated in our institution in the last past two years, were retrospectively included in this study. The cohort consists in forty-five lesions treated for lung SBRT using the MidP technique.

\section{Respiration-correlated 4D-CT imaging}

All patients underwent a planning imaging session on a big bore CT scanner (Aquilion LB, Canon medical systems corporation, Japan). For the acquisitions, patients were immobilized in supine position using half body vacuum bag (Orfit Industries, Belgium) with arms raised above the head. A $4 \mathrm{D}-\mathrm{CT}$ was acquired with patients undergoing audio-coaching to regularize their breathing with the aim of reducing 4D-CT image artefacts [17]. In this acquisition mode, the CT scanner automatically sets the optimal helical pitch according to the patient's breathing period measured using the GateCT surface guided system (Vision RT Ltd, UK). The 4D-CT datasets were reconstructed with a $2 \mathrm{~mm}$ slice thickness and were sorted in a phase-based process into 10 respiratory phases ( $0 \%$ representing endinhalation and $50 \%$ representing end-exhalation). Finally, an average CT was computed using all 10 image sets. Those data sets were used to delineate a GTV(s) and generate a PTV for each of the different motion management strategies.

\section{Definition of target volumes}

Gross tumor volume (GTV) were delineated using the lung window setting ( $\mathrm{L}=600 \mathrm{HU}, \mathrm{W}=1600 \mathrm{HU}$ ). This was done by the same expert radiation oncologist in order to minimize the tumor contour variability between CTs.

\section{Internal target volume}

The union of all the GTV delineated on each of the 4D-CT phases give rise to the individual ITV.

\section{Mid-position}

Target definition was performed on the artificial mid-position CT generated by the open-source OpenReggui software[18]. It provides a framework to generate, by means of deformable image registration, a 3D mid-position CT using a 4DCT set as input. 
1 - Manual delineation on a single phase

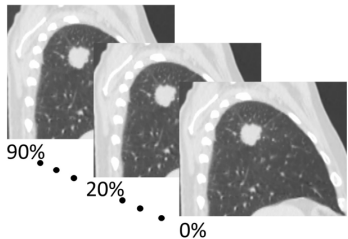

3 - Identification of the mid-ventilation (MidV) phase

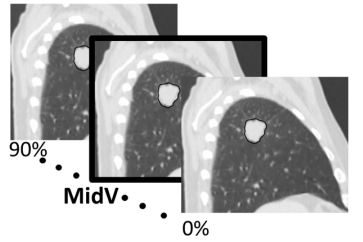

4 - Calculation of $\sigma_{T M}$ based on tumor's Center of mass motion

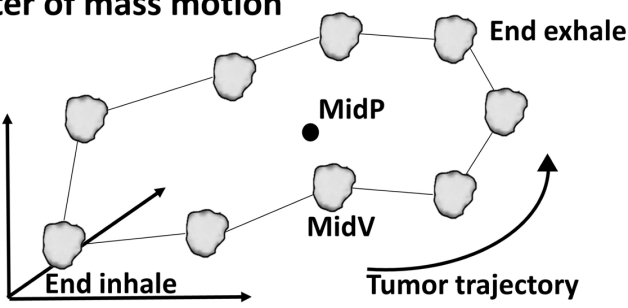

2 - Deformable registration and ROI mapping

(visual inspection and manual editing if necessary)

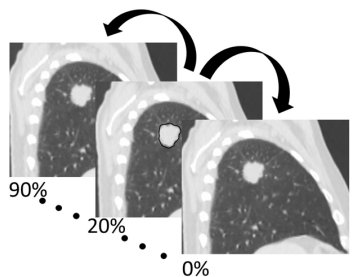

3.5 - Translation of the MidV_GTV at the midposition coordinates (MidP)

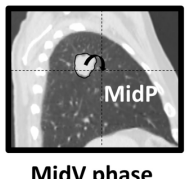

Unique to the Hybrid MidV-MidP workflow

\section{5 - GTV expansion and PTV creation on the average CT}

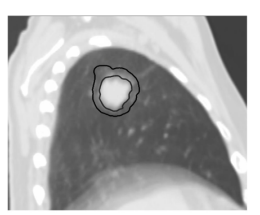

Figure 1 - Schematic description of the script workflow for the creation of MidV and Hybrid MidV-MidP PTVs. Steps 2 to 5 are completely automated by the script. Step 1 is a prerequisite for starting the script. Step 2 eases the physician work in mapping the delineated GTV from step 1 based on deformable registration results with the other 4D-CT phases. Step 3 illustrates the selection of the mid-ventilation (MidV) phase as the closest to the time-weighted mean position of the tumor (Mid P). Step 3.5 is related to the Hybrid MidV-MidP workflow where the MidV_GTV is translated at the MidP coordinates. Step 4 illustrates the GTV evolution through the respiratory cycle of a theoretical patient presenting a typical hysteresis-like tumor motion. Step 5 illustrate the creation of the MidV_PTV using anisotropic margins that account for the calculated tumor motion.

\section{Mid-ventilation}

Target definition was performed on the mid-ventilation phase. This phase was identified using a homemade RayStation treatment planning script (clinical version 9B, RaySearch Laboratories, Stockholm, Sweden) using IronPython v2.7. It was implemented to fully integrate the MidV workflow into the treatment planning system. Figure 1 shows the script's workflow (publically available under the "Hybrid MidV-MidP" bitbucket.org repository). The MidV-CT was calculated as the phase where the GTV position minimize the 3D vector amplitude to the mid-position. It thus requires the GTV to be delineated on each of the 4DCT phases. To reduce this burden, the script features an automated use of DIR (cf. Figure 1-step 1) and structure mapping (cf. Figure 1-step 2) to propagate a single GTV contours to the rest of the $4 D C T$. The delineated phase was standardized as the $20 \%$ for its likelihood to be selected as the MidV-CT[12]. While mandatory, this step minimizes the need for contouring and reduces the intra-observer variabilities. The resulting contours on each phase were reviewed and corrected (if necessary) by the radiation oncologist as prompted by the script. After tumor trajectory 
analysis, the mid-ventilation frame was selected and renamed for authentication purposes (cf. Figure 1-step 3).

\section{Hybrid MidV-MidP}

A refinement of the mid-ventilation approach is given by translating the mid-ventilation GTV onto the mid-position coordinates based on its center of mass (Figure 1-step 3.5). This 3D structure manipulation was implemented as an additional step in the mid-ventilation workflow. For the sake of simplicity, it is referred as "Hybrid MidV-MidP" as the GTV is defined on the MidV phase and the PTV is created at the MidP coordinates.

\section{Margin definition}

Margins definition is based on the formalism proposed by Van Herk et al. [19]. As there is no CTV extension for lung SBRT (i.e., CTV = GTV)[20], the GTV-to-PTV margin recipe is formulated, using the exact same terminology as Wanet et al.[14], as:

$$
M_{M i d P}=2.5 \times \sqrt{\Sigma_{T M}^{2}+\Sigma_{B L}^{2}+\Sigma_{S E T U P}^{2}+\Sigma_{D}^{2}}+1.64 \times \sqrt{\sigma_{T M}^{2}+\sigma_{B L}^{2}+\sigma_{S E T U P}^{2}+\sigma_{p}^{2}}-1.64 \sigma_{p}
$$

where $\Sigma$ and $\sigma$ denote the standard deviations of the systematic and random errors, respectively. Subscripts $T M, B L, S E T U P, D$ and $p$ refer to tumor motion, baseline shift, patient setup variability, delineation uncertainty, and penumbra respectively. The margin formula aims at obtaining $95 \%$ minimum dose coverage for $90 \%$ of the patients when all geometric uncertainties are included. All standard deviations were set in agreement with the published literature $[2,12,21]$ with the exception of $\sum_{T M}$ and $\sigma_{T M}$. These values were similar for the MidP, MidV, Hybrid MidV-MidP and ITV strategies. In the particular case of the penumbra, its width $\sigma_{p}$ for our Elekta Infinity (Elekta $A B$, Sweden) was computed in an identical manner as previously published by Wanet et al.[14] for the Tomotherapy machines (Accuray, Sunnyvale, CA): the parameter was modeled by the standard deviation of a cumulative Gaussian fitted to the calculated dose profiles. In lung where the penumbra is larger than in water, $\sigma_{p}$ was computed to be $5.5 \mathrm{~mm}$ in each direction. Finally, standard deviations related to tumor motion were estimated on an individual basis, depending on the considered margin strategy. For the MidP, $\sum_{T M}$ was fixed to $0.5 \mathrm{~mm}$ which corresponds to the residual error of deformable registration[18] while it was set to 0 for all the other strategies. For the MidP, MidV and Hybrid MidV-MidP, $\sigma_{T M}$ was calculated for each patient individually using the tumor motion amplitude measured in the 4D-CT along each axis[12,13]. Unique to the MidV approach, an error term $\varepsilon_{\text {MidV-MidP }}$ was linearly added to the margin recipe to compensate for the inherent distance between mid-ventilation and mid-positon locations. By construction, as the ITV accounts for tumor motion-related uncertainties, both $\sum_{T M}$ and $\sigma_{T M}$ were set to $0 \mathrm{~mm}[13]$.

Spatial and volumetric comparisons of the resulting volumes were performed and statistical significance was assessed using a Wilcoxon's signed-rank test with a 0.05 significance level ( $p$-value). 


\section{Results}

\section{Patients and tumors characteristics}

Lesions characteristics are reported in Table 1. The majority of patient had peripheral tumor (71\%) predominantly localized in the upper lobe (64\%).

Table 1 - Distribution of lesions characteristics by location.

\begin{tabular}{llll}
\hline & Central tumor & Peripheral tumor & Total group \\
\hline Upper Lobe & 8 & 21 & 29 \\
Lower Lobe & 4 & 9 & 13 \\
Middle lobe & 1 & 2 & 3 \\
\cline { 2 - 4 } $\begin{array}{l}\text { Total } \\
\text { Average (std. dev.) }\end{array}$ & & 32 & 45 \\
\hline GTV Volume on MidP [cc] & $3.4(2.2)$ & $4.8(5.6)$ & $4.4(4.9)$ \\
3D Motion amplitude [mm] & $13(7)$ & $9(5)$ & $10(6)$ \\
\hline
\end{tabular}

Script validation

The script was initially validated using the dynamic thorax phantom (Computerized Imaging Reference Systems, Inc., VA, USA) with a known tumor motion amplitude of $(1,1,2) \mathrm{cm}$ in the (rightleft, anterior-posterior, superior-inferior) directions respectively. The GTV's center of mass coordinates on each of the 4D-CT phases was manually collected. The resulting set of coordinates serves to independently crosscheck each parameters calculated by the script. Reviewed parameters includes both MidV and MidP coordinates, identification of the MidV-phase, tumor motion and margin amplitude evaluation. The same validation procedure was repeated for each of the 45 lesions individually. No differences are observed between the script and manual calculation.

Spatial and volumetric comparison

Owing to hysteresis-like motion, mid-ventilation selection using the three-dimensional tumor motion had an average (SD) of $1.1(0.9) \mathrm{mm}$ tumor centroid proximity with respect to the mean tumor position.

Figure 2 shows the margin expansion for the different motion management strategies in the anterior-posterior (AP), superior-inferior (SI) and right-left (RL) directions (uncoupled for the MidV case). As the linear addition of the $\varepsilon_{\text {MidV-MidP }}$ component to the MidV margin recipe is directional, it was only found significantly larger $(p<0.03)$ than the MidP in the posterior direction by an average of $+0.3 \mathrm{~mm}$. While negligible at the PTV margin level, the Hybrid MidV-MidP margin was found to be statistically smaller $(p<0.01)$ than the MidP in the RL $(-0.2 \mathrm{~mm})$ and AP $(-0.1 \mathrm{~mm})$ directions. It is hypothesized that OppenReggui slightly underestimates the tumor motion due to the grid regularization term trying to maintain a diffeomorphic transformation. It results in a lower calculated margin in the direction presenting the largest tumor motion amplitude (i.e SI). 
By construction, the Hybrid MidV-MidP margin was also found smaller than the MidV in every directions by $(-0.2,-0.1),(-0.2,-0.3)$ and $(-0.5,-0.3) \mathrm{mm}$ in $(R, L),(A, P)$ and $(S, I)$ respectively $(p<0.01)$.

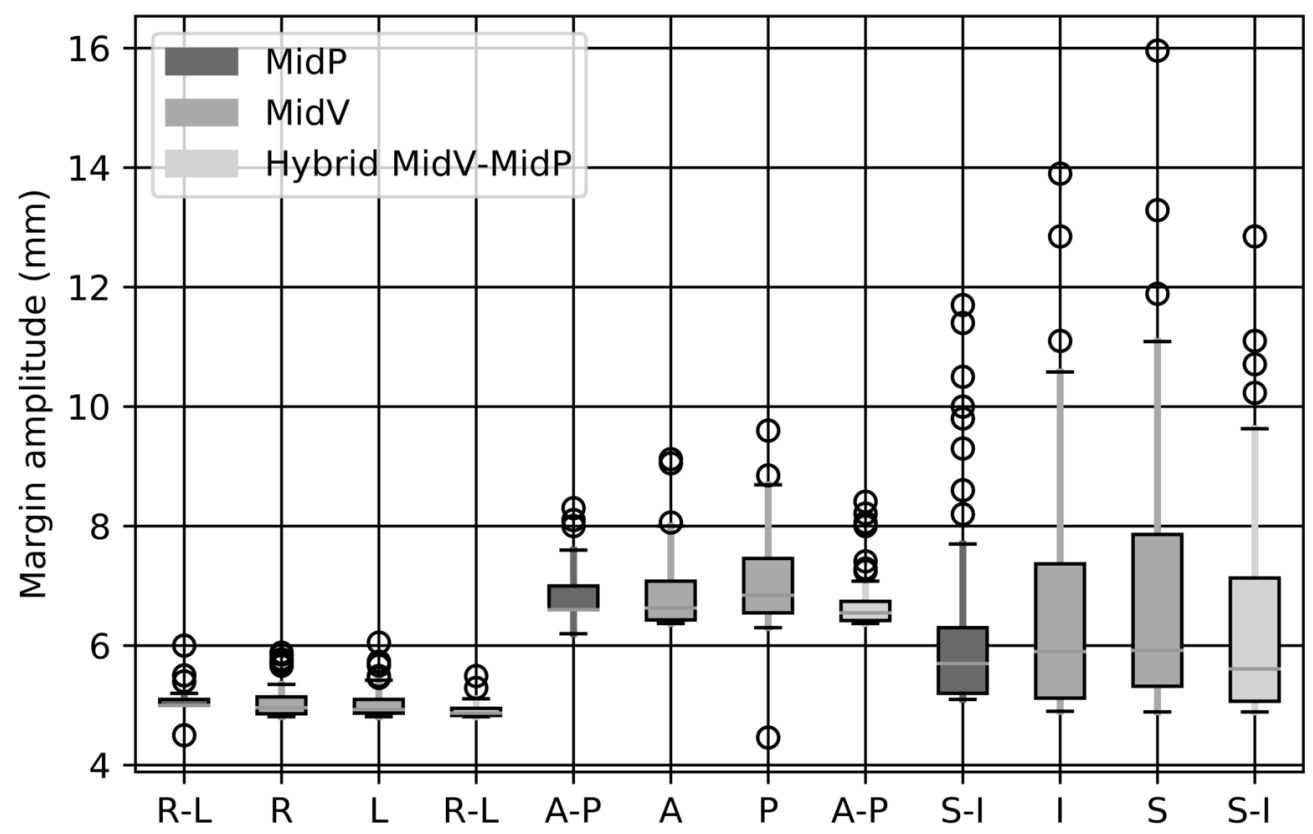

Figure 2 - PTV margin calculated for all the different 4D workflow in the three spatial directions (uncoupled for the MidV case). Outliers were defined as points outside the range defined by [Q1-1.5(IQR); Q3+1.5(IQR)], where Q represents the quartile and IQR the interquartile range.

The resulting planning target volume are presented for each PTV strategy in Figure 3. As expected, the ITV showed significantly larger target volume $(p<0.01)$ with an average of $+5.7(4.2) c c,+5.5(4.3)$ and $+6.4(4.7) \mathrm{cc}$ from the MidP, MidV and Hybrid strategies respectively. No statistical differences were found between MidV and MidP PTV volume. By construction, the Hybrid MidV-MidP PTV exhibited the smallest volume and was on average $26(7.1) \%, 4.7(4.6) \%$ and $2.3(9.7) \%$ smaller than the ITV, MidV and MidP generated PTVs respectively $(p<0.014)$.

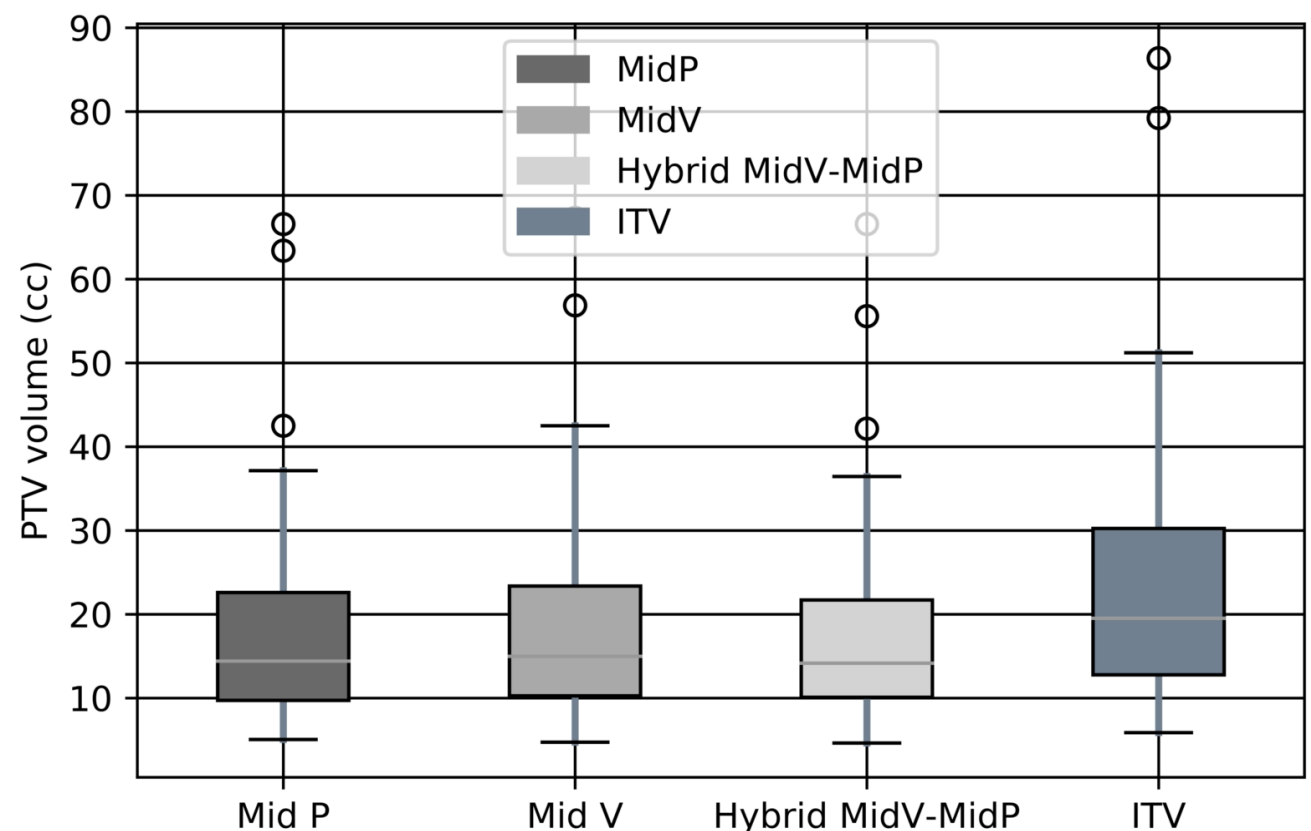


Figure 3 - PTV volume generated using the different treatment planning-based motion management strategies. Outliers were defined as points outside the range defined by [Q1-1.5(IQR); Q3+1.5(IQR)], where Q represents the quartile and IQR the interquartile range.

The margin in the AP direction was slightly larger than in the SI and RL directions due to a more important contribution of intra-fraction variability (cf. Figure 4). The quadratic dependence of the Hybrid MidV-MidP margin on respiratory motion was only modestly influenced by the peak-to-peak tumor amplitude of up to $10 \mathrm{~mm}$.

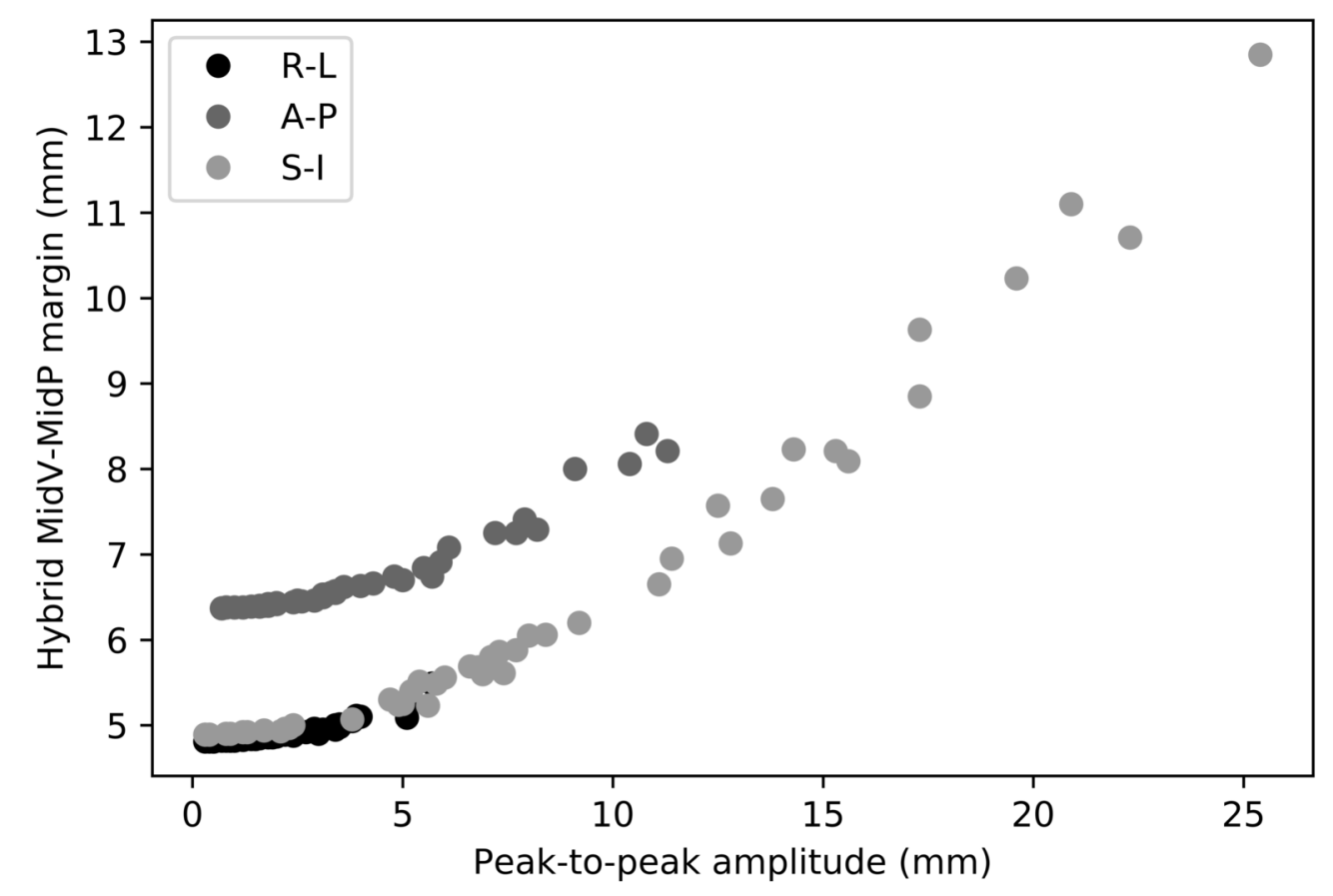

Figure 4 - Dependence of the Hybrid MidV-MidP margin recipe to the tumor motion in the three spatial directions.

\section{Discussion}

With favorable published clinical outcomes[22], both MidP and MidV strategies have proven to be reliable solutions to incorporate motion information from 4D-CT scans into the treatment planning process. Their introduction in clinical routine might be challenging as all published methods rely on in-house software solutions[18] or on a combination of close and open source software[23].

Herewith, we report on the successful integration of a MidV-based motion management technique into the treatment planning system using built-in scripting functionalities. It allows for the selection of an appropriate CT scan from the 4D data set where the tumor is the closest to its mean position in the respiratory cycle. Our MidV workflow uses deformable image registration (DIR) to ease the radiation oncologist's work: the GTV delineated on a single phase is propagated to the rest of the 4D$\mathrm{CT}$ and the resulting contours are manually reviewed. The implementation of a MidV workflow does not necessarily require the use of DIR but it was found to improve delineation consistency[24]. Compared to the MidP, the implication of DIR is limited, as it is not used to generate a new CT. Based on previous MidP knowledge, the MidV margin recipe is adapted by linearly adding a directional component $\varepsilon_{\text {MidV-MidP }}$ with the amplitude of the corresponding vector linking the MidP to the MidV 
coordinates. In our patient cohort, the average amplitude of this 3D vector is $1.1(0.9) \mathrm{mm}$ which is in agreement with the 1.3(0.9) published in the literature[25]. It thus contributes modestly to the total margin. However, the margin amplitude shows an already described[16] quadratic behavior with tumor peak-to-peak amplitude, leading to an important dependence of exact target motion evaluation for a third of the case analyzed (directional motion $>15 \mathrm{~mm}$ ).

Even though not statistically significant, the MidV approach tends, in average, towards slightly larger margins in the $S(+0.3 \mathrm{~mm}), I(+0.2 \mathrm{~mm})$ and $P(+0.3 \mathrm{~mm})$ directions. Whereas in $R, L$ and $A$ directions, the impact of the deformable registration systematic error $\sum_{T M}$ on the MidP margin recipe is balanced by the directional $\varepsilon_{\text {MidV-MidP }}$ component in the MidV recipe. Hence, the gain in using the MidP in comparison to the MidV is rather limited when the coregistration uncertainty is taken into account in the margin recipe.

The MidV strategy is refined using the MidP-based information by translating the GTV_MidV at the MidP coordinates (i.e. Hybrid MidV-MidP). The resulting PTV volumes were on average 26(7.1)\%, 4.7(4.6)\% smaller than the ITV and MidV respectively (cf. Figure 5). Those results are similar to the reported values from other studies[10,14] comparing the MidP and MidV approaches to the ITV. The Hybrid MidV-MidP offers a substantial reduction in the actual irradiated volume compared to the ITV that might allow for better organ sparing or leave space for dose escalation[26]. When compared to the MidV strategy, the margin reduction gain is only relevant for tumor presenting large hysteresis motion and can results in a reduction of the irradiated volume up to $17 \% / 4.5 \mathrm{cc}$.

According to the recent ESTRO-ACROP guidelines[20], the ITV is still the most commonly used strategy to treat lung SBRT lesions rather than the MidP or MidV strategies. There might be several reasons for this. The ITV is a safe approach for ensuring target coverage that relies on less hypothesis than the MidP/midV concept. For instance, in cases with extreme motion directionality (e.g. close to the $45^{\circ}$ in the orthonormal coordinate system), the ITV might supersede the MidP/MidV concept. However, the MidP concept has yielded satisfactory target coverage in numerous studies [10,14-16]. The MidP concept can accommodate to some extend irregular tumor motion, due to the central limit theorem, as long as the variance is not affected too much. Exceptions appear when simplifications used to formulate the margin recipe model are no longer valid. The regular respiratory motion is well represented by the asymmetric $\sin ^{4}$ function[27] and differs from normality. Hence the total random error deviates from a Gaussian distribution predicted by the central limit theorem when the breathing motion amplitude is large compared to the other sources of uncertainty[28]. In addition, the aspherical shape of the GTV can only be ignored if its size is large compared to the various standard deviations[19]. Otherwise, both Wanet et al.[14] and Bellec et al.[10] showed a loss of target coverage for small lesions presenting large motion amplitudes. On top of the already small margins used in SBRT, the additional reduction inferred by the use of MidV/MidP strategy might discourage their introduction in clinical routine. Furthermore, there is currently no commercial solution providing a framework that would allow departments either to determine the midventilation phase or to generate a mid-position image from the 4D-CT data. This, in itself, strongly impedes on the widespread adoption of the MidP and MidV strategies in clinical routine, resulting in larger irradiated volumes than would be necessary. As stated by several authors [29,30], the optimal PTV should be defined as the volume that maintains the smallest margins without sacrificing target coverage. Hence, the actual uncertainties measured should be incorporated into the margin recipe when no active motion management technique is available. To our knowledge, we are the first to 
develop a fully TPS-integrated solution for 4D-CT MidV-based planning. Even though the results are provided for lung SBRT patients, the concept can be transposed to any moving tumor. It could serve as a basis for the development of new features in both commercial treatment planning system and CT-scanner software.

The selected mid-ventilation phase (on average the $20 \%$ for exhalation and $70 \%$ for inhalation) are presenting a rapid tumor motion. This high velocity increases the risk for breathing-induced motion artifacts in 4D CT imaging. Such artifacts may influence the volume, shape, and even "position" of pulmonary lesions, resulting in systematic errors in treatment planning. While blurrier than the $\mathrm{MidP}-\mathrm{CT}$, the breathing-induced motion artefacts in the mid-ventilation frames have proven to cause negligible changes in the delineated tumor volume and shape [25]. In a consistent manner, no statistically significant differences were found between the GTV volumes delineated on 4D-CT phases and those delineated in MidP-CT. Our data confirm that the resulting PTV volume is therefore only influenced by the margin recipe components. On the level of organ-at-risk, 4DCT phases are noisier than conventional CT since the exposure per frame is lower. Additionally, they might contain artefacts due to breathing irregularities. Hence, the MidP-CT is best suited for moving organ-at-risk delineation as it represents all the anatomical structures at their mean position in the respiratory cycle.

The current study focuses on the feasibility of integrating, into the TPS, a 4DCT planning workflow that generates a PTV volume comparable to the MidP one using individualized margins. Therefore, we did not investigated the dosimetric impact of a reduction in the PTV volume. This was already described by Wanet et al., who compared the dose received by organs-at-risk when planning lung SBRT using both the MidP and ITV concept. Jin P. et al. [31] also performed this comparison for neoadjuvant radiotherapy of esophageal cancer. Velec $\mathrm{M}$ et al. [26] investigated the place of dose escalation for liver SBRT using a PTV defined at the mean respiratory position. Although these studies showed a dose reduction for organs at risk, it is difficult to infer if it leads to a clinical benefit. However, it is fair to state that a dose reduction to OARs can result in a lower incidence of certain clinical endpoints[32]. Consequently, the statistically significant reduction in irradiated volume in the present study comparing the ITV, MidV and MidP strategies might be considered as being potentially clinically relevant.

Finally, MidP users should weight the benefits provided by the motion compensated CT with the benefits of a TPS-embedded solution. They should stay vigilant and be able to assess the DIR quality as its use on highly mobile tumors, sliding along the chest wall, might be challenging[33].

\section{Conclusion}

A novel strategy, that incorporates the tumor motion into the margin recipe, was developed by translating the mid-ventilation GTV onto the mid-position coordinates (e.g. Hybrid MidV-MidP). It results in comparable irradiated volumes as the MidP strategy while being independent from any deformable registration performance. We showed the feasibility of integrating both MidV and Hybrid MidV-MidP strategies into the treatment planning system. It answers the need for a 4DCT treatment planning workflow, integrated in a controlled environment, with limited radiation oncologist intervention. 


\section{Conflict of interest}

The authors declare they have no conflict of interest.

\section{Bibliography}

[1] Prescribing, Recording, and Reporting Photon-Beam Intensity-Modulated Radiation Therapy (IMRT): J ICRU 2010;10:NP.3-NP. https://doi.org/10.1093/jicru/ndq002.

[2] Bissonnette JP, Franks KN, Purdie TG, Moseley DJ, Sonke JJ, Jaffray DA, et al. Quantifying Interfraction and Intrafraction Tumor Motion in Lung Stereotactic Body Radiotherapy Using Respiration-Correlated Cone Beam Computed Tomography. Int J Radiat Oncol Biol Phys 2009;75:688-95. https://doi.org/10.1016/j.ijrobp.2008.11.066.

[3] Schwarz M, Alber M, Lebesque J V., Mijnheer BJ, Damen EMF. Dose heterogeneity in the target volume and intensity-modulated radiotherapy to escalate the dose in the treatment of non-small-cell lung cancer. Int J Radiat Oncol Biol Phys 2005;62:561-70. https://doi.org/10.1016/j.ijrobp.2005.02.011.

[4] S S Vedam, P J Keall, V R Kini, H Mostafavi HPS and RM. Acquiring a fourdimensional computed tomography dataset using an external respiratory signal. Phys Med Biol 2002;48. https://doi.org/https://doi.org/10.1088/0031-9155/48/1/304.

[5] Ford EC, Mageras GS, Yorke E LC. Respiration-correlated spiral CT: a method of measuring respiratory-induced anatomic motion for radiation treatment planning. Med Phys 2003;30:8897. https://doi.org/10.1118/1.1531177.

[6] Keall PJ, Starkschall G, Shukla H, Forster KM, Ortiz V, Stevens CW, Vedam SS, George R, Guerrero T MR. Acquiring 4D thoracic CT scans using a multislice helical method. Phys Med Biol 2004;49:20053-67.

[7] Pan T, Lee TY, Rietzel E CG. 4D-CT imaging of a volume influenced by respiratory motion on multi-slice CT. Med Phys 2004;31:333-40. https://doi.org/10.1118/1.1639993.

[8] Engelsman M, Damen E, De Jaeger K, van Ingen KM, Mijnheer BJ. The effect of breathing and set-up errors on the cumulative dose to a lung tumor. Radiol Oncol 2001;60:95-105.

[9] Witte MG, van der Geer J, Schneider C, Lebesque JV van HM. The effects of target size and tissue density on the minimum margin required for random errors. Med Phys 2004;31:306879. https://doi.org/10.1118/1.1809991.

[10] Bellec J, Arab-Ceschia F, Castelli J, Lafond C, Chajon E. ITV versus mid-ventilation for treatment planning in lung SBRT: A comparison of target coverage and PTV adequacy by using in-treatment 4D cone beam CT. Radiat Oncol 2020;15:1-10. https://doi.org/10.1186/s13014-020-01496-5.

[11] Thengumpallil S, Racine D, Germond J, Péguret N, Bourhis J, Bochud F, et al. Retrospective analysis of the impact of respiratory motion in treatment margins for frameless lung SBRT based on respiratory-correlated CBCT data-sets. J Appl Clin Med Phys 2020:1-9. https://doi.org/10.1002/acm2.13034.

[12] Wolthaus, Jochem W H, Schneider C, Sonke JJ, Van Herk, Marcel, Belderbos, Jose S A, Rossi, Maddalena M G, Lebesque, Joos V, Damen EMF. Mid-ventilation CT scan construction from four-dimensional respiration-correlated CT scancs for radiotherapy planning of lung cancer patients 2006;65:1560-71. https://doi.org/10.1016/j.ijrobp.2006.04.031.

[13] Wolthaus JWH, Sonke JJ, van Herk M, Belderbos JSA, Rossi MMG, Lebesque J V., et al. Comparison of Different Strategies to Use Four-Dimensional Computed Tomography in Treatment Planning for Lung Cancer Patients. Int J Radiat Oncol Biol Phys 2008;70:1229-38. 
https://doi.org/10.1016/j.ijrobp.2007.11.042.

[14] Wanet M, Sterpin E, Janssens G, Delor A, Lee JA, Geets X. Validation of the mid-position strategy for lung tumors in helical TomoTherapy. Radiother Oncol 2014;110:529-37. https://doi.org/10.1016/j.radonc.2013.10.025.

[15] Thomas SJ, Evans BJ, Harihar L, Chantler HJ, Martin AGR, Harden S V. An evaluation of the mid-ventilation method for the planning of stereotactic lung plans. Radiother Oncol 2019;137:110-6. https://doi.org/10.1016/j.radonc.2019.04.031.

[16] Peulen H, Belderbos J, Rossi M, Sonke J-J. Mid-ventilation based PTV margins in stereotactic Body Radiotherapy (SBRT): clinical evaluation. Radiother. Oncol., DE GRUYTER; 2014, p. 511-6. https://doi.org/10.1515/9783110289039.619.

[17] Goossens S, Senny F, Lee JA, Janssens G, Geets X. Assessment of tumor motion reproducibility with audio-visual coaching through successive 4D CT sessions. J Appl Clin Med Phys 2014;15:47-56. https://doi.org/10.1120/jacmp.v15i1.4332.

[18] Janssens G. Registration models for tracking organs and tumors in highly deformable anatomies : applications to radiotherapy. UNIVERSITE CATHOLIQUE DE LOUVAIN, 2010. https://doi.org/http://hdl.handle.net/2078.1/33459.

[19] Van Herk M, Remeijer P, Rasch C, Lebesque J. The probability of correct target dosage: dosepopulation histogrames for deriving treatment margins in radiotherapy. Int J Radiat Oncol Biol Phys 2000;47:1121-35.

[20] Guckenberger M, Andratschke N, Dieckmann K, Hoogeman MS, Hoyer M, Hurkmans C, et al. ESTRO ACROP consensus guideline on implementation and practice of stereotactic body radiotherapy for peripherally located early stage non-small cell lung cancer. Radiother Oncol 2017;124:11-7. https://doi.org/10.1016/j.radonc.2017.05.012.

[21] Sonke J-J, Lebesque J, van Herk M. Variability of four-dimensional computed tomography patient models. Int J Radiat Oncol Biol Phys 2008;70:590-8. https://doi.org/10.1016/j.ijrobp.2007.08.067.

[22] Peulen H, Belderbos J, Rossi M, Sonke J. Mid-ventilation based PTV margins in Stereotactic Body Radiotherapy ( SBRT ): A clinical evaluation. Radiother Oncol 2014;110:511-6. https://doi.org/10.1016/j.radonc.2014.01.010.

[23] Van Herk M, McWilliam A, Whitehurst P, Faivre-Finn C. EP-1844: Feasibility of generating mid-position CT from 4DCT using commercial deformable registration systems. Radiother Oncol 2016;119:S867-8. https://doi.org/10.1016/s0167-8140(16)33095-x.

[24] Vinod SK, Min M, Jameson MG, Holloway LC. A review of interventions to reduce interobserver variability in volume delineation in radiation oncology. J Med Imaging Radiat Oncol 2016;60:393-406. https://doi.org/10.1111/1754-9485.12462.

[25] Guckenberger M, Ilbert J, Rieger T, Ichter A, Aier K, Lentje M. Mid-ventilation concept for mobile pulmonary tumors: internal tumor trahectory versus selective reconstruction of fourdimensional computed tomography frames based on external breathing motion 2009;74:602-9. https://doi.org/10.1016/j.ijrobp.2008.12.062.

[26] M V, J.L M, L.A D, K.K B. Dose-escalated liver stereotactic body radiotherapy (SBRT) at the mean respiratory position. Int J Radiat Oncol Biol Phys 2014;89:1121-8. https://doi.org/10.1038/jid.2014.371.

[27] Lujan AE, Larsen EW, Balter JM, Haken RK Ten. A method for incorporating organ motion due to breathing into 3D dose calculations. Med Phys 1999;26:715-20.

[28] Van Herk M, Witte M, Van Der Geer J, Schneider C, Lebesque J V. Biologic and physical fractionation effects of random geometric errors. Int J Radiat Oncol Biol Phys 2003;57:146071. https://doi.org/10.1016/j.ijrobp.2003.08.026.

[29] Aznar MC, Warren S, Hoogeman M, Josipovic M. The impact of technology on the changing practice of lung SBRT. Phys Medica 2018;47:129-38. 
https://doi.org/10.1016/j.ejmp.2017.12.020.

[30] Yang M, Timmerman R. Stereotactic Ablative Radiotherapy Uncertainties: Delineation, Setup and Motion. Semin Radiat Oncol 2018;28:207-17.

https://doi.org/10.1016/j.semradonc.2018.02.006.

[31] Jin P, Machiels M, Crama KF, Visser J, van Wieringen N, Bel A, et al. Dosimetric Benefits of Midposition Compared With Internal Target Volume Strategy for Esophageal Cancer Radiation Therapy. Int J Radiat Oncol Biol Phys 2019;103:491-502. https://doi.org/10.1016/j.ijrobp.2018.09.024.

[32] Mulryan K, Leech M, Forde E. Effect of stereotactic dosimetric end points on overall survival for Stage I non-small cell lung cancer: A critical review. Med Dosim 2015;40:340-6. https://doi.org/10.1016/j.meddos.2015.04.005.

[33] Sarrut D, Baudier T, Ayadi M, Tanguy R, Rit S. Deformable image registration applied to lung SBRT: Usefulness and limitations. Phys Medica 2017;44:108-12. https://doi.org/10.1016/j.ejmp.2017.09.121. 